\title{
Human cystic and alveolar echinococcosis in the Tibet Autonomous Region (TAR), China
}

\author{
X. Feng ${ }^{1,4}$, X. Qi ${ }^{1}$, L. Yang ${ }^{1}$, X. Duan ${ }^{1}$, B. Fang ${ }^{1}$, Q. Gongsang ${ }^{2}$, \\ B. Bartholomot ${ }^{3}$, D.A. Vuitton ${ }^{3}$, H. Wen ${ }^{1 *}$ and P.S. Craig ${ }^{4 *}$ \\ ${ }^{1}$ Xinjiang Hydatid Clinical Research Institute, The First Affiliated Hospital \\ of Xinjiang Medical University, No.137 Liyushan South RD, Urumqi \\ 830054, Xinjiang, P. R. China: ${ }^{2}$ Centre of Disease Prevention and Control, \\ No. 21 Linkuo North RD, Lhasa, Tibet Autonomous Region: \\ ${ }^{3}$ WHO Collaborating Centre for Prevention and Treatment of Human \\ Echinococcosis, SERF Research Unit EA 2276, University of Franche- \\ Comte, 25030 Besancon Cedex, France: ${ }^{4}$ Cestode Zoonoses Research \\ Group, School of Environment and Life Sciences, University of Salford, \\ M5 4WT, UK
}

(Received 27 March 2015; Accepted 3 July 2015; First Published Online 14 August 2015)

\begin{abstract}
Human cystic echinococcosis (CE) is known to be endemic in the Tibet Autonomous Region (TAR), China; however, there is relatively little data from hospital records or community prevalence studies, and the situation regarding occurrence of human alveolar echinococcosis (AE) is unclear. Here we review the available reports about human echinococcosis in the seven prefectures of TAR. In addition, two pilot studies by mass screening using ultrasound (with serology) were undertaken (2006/7) in Dangxiong County of Lhasa Prefecture (north central TAR) and Dingqing County of Changdu Prefecture (eastern TAR). In Dangxiong County a prevalence of $9.9 \%(55 / 557)$ for human CE was obtained but no human AE cases were detected. By contrast, in Dingqing County ( $N=232$ persons screened), $11 \mathrm{CE}$ cases (4.7\%) and $12 \mathrm{AE}$ cases (5.2\%) (including one mixed $\mathrm{CE}$ and $\mathrm{AE}$ case) were diagnosed by ultrasound. Hospital records and published reports indicated that CE cases were recorded in all of seven prefectures in Tibet Autonomous Region, and AE cases in four prefectures. Incidence rates of human CE were estimated to range from 1.9 to 155 per 100,000 across the seven prefectures of TAR, with a regional incidence of 45.1 per 100,000. Incidence of AE was estimated to be between 0.6 and 2.8 cases per 100,000. Overall for TAR, human AE prevalence appeared relatively low; however, the pilot mass screening in Dingqing in eastern TAR indicated that human AE disease is a potential public health problem, possibly similar to that already well described in Tibetan communities bordering TAR in north-west Sichuan and south-west Qinghai provinces.
\end{abstract}

\section{Introduction}

Human cystic echinococcosis (CE) and alveolar echinococcosis (AE) are helminthic zoonotic diseases caused by infection with the metacestode stages of 
Echinococcus granulosus (sensu lato) and Echinococcus multilocularis, respectively. Both are highly endemic in north-western China and are an important public health problem (Jiang, 2002; Craig 2004; Ministry of Health, 2005). A national echinococcosis control programme was implemented from 2006/7 in western China with emphasis on dog deworming, but has not yet been rolled out effectively in the more remote communities in western China, including north-west Xinjiang and much of the Tibet Autonomous Region (TAR) (Craig et al., 2007, 2008; van Kesteren et al., 2015). The burden of both human $\mathrm{CE}$ and $\mathrm{AE}$ disease is known to be particularly high in Tibetan communities located in north-west Sichuan and south-west Qinghai provinces on the eastern Tibetan plateau (Budke et al., 2005; Li et al., 2005, 2010).

The Tibet Autonomous Region covers 1.23 million $\mathrm{km}^{2}$ and is bordered to the north by Qinghai Province and Xinjiang Uygur Autonomous Region, to the east by Sichuan and Yunnan provinces, and to the south with Myanmar, India, Bhutan and Nepal. The TAR (capital Lhasa) forms a major part (47\%) of the Qinghai-Tibetan Plateau (total area 2.5 million $\mathrm{km}^{2}$ ). With an average elevation over $4000 \mathrm{~m}$ and a population of 2.81 million, TAR has the lowest population density (2.3 inhabitants per $\mathrm{km}^{2}$ ) in China (National Bureau of Statistics of China, 2007). TAR is divided into one prefecture-level city (Lhasa) and six prefectures: Naqu (Nagqu), Ali (Ngari), Linzhi (Nyinchi), Changdu (Qamdo), Shannan and Rikaze (Xigazê). In addition, a significant Tibetan population is present in the high pasture regions of four other provinces of China (i.e. Qinghai, Sichuan, Gansu and Yunnan) with a total Tibetan population in China of approximately 6 million, including TAR.

Human cystic echinococcosis (CE) has been formally recognized as endemic in the TAR since 1987 (Hu et al., 1987) and CE cases have been found continuously among the predominant nomadic pastoral population on the Tibetan plateau outside the TAR (in the neighbouring provinces of Sichuan, Qinghai and Gansu). Mass screening programmes for human echinococcosis, principally using portable ultrasound to detect abdominal $\mathrm{CE}$ or $\mathrm{AE}$, have been undertaken in several Tibetan Autonomous Counties and Prefectures outside the TAR; particularly in Qinghai and Gansu provinces and in western Sichuan Province (Bai et al., 2002; Schantz et al., 2003; Li et al., 2010). These studies revealed a very high prevalence of both $\mathrm{CE}$ and $\mathrm{AE}$ in Tibetan communities in Sichuan and Qinghai, with mean prevalences of $4-6 \%$ (although the prevalence at the township level ranges from 1 to $15 \%$ ) for both $\mathrm{CE}$ and $\mathrm{AE}$ (Li et al., 2010). In 2004, the Chinese Ministry of Health carried out a nationwide assessment of eight parasitic diseases, including echinococcosis (Ministry of Health, 2005). The highest overall ultrasound CE prevalence $(2.4 \%)$ by ethnicity was in the Tibetan communities of TAR, north-west Sichuan and Qinghai Province. Despite these studies, there remains a relative lack of information regarding the prevalence and public health burden of echinococcosis (CE and AE) in the TAR.

In order to investigate the status of human echinococcosis in TAR, we reviewed published reports on echinococcosis in the TAR up to 2006/7, and also patient records from the People's Hospital of Changdu Prefecture in eastern TAR. In addition, we carried out pilot community studies based on mass ultrasound screening in Dangxiong County of Lhasa Prefecture and Dingqing County of Changdu Prefecture (eastern TAR).

\section{Materials and methods}

\section{Study sites}

In 2006 and 2007 two communities (one each in Dangxiong and Dingqing counties) in TAR (fig. 1) were investigated by mass screening for abdominal echinococcosis, and in addition hospital records in the People's Hospital of Changdu Prefecture were reviewed for human echinococcosis cases.

Dangxiong County is situated in the north of Lhasa Prefecture, adjoining Naqu Prefecture. Human CE cases have previously been reported from the county (Gong et al., 2001). Dangxiong County covers an area of $10,036 \mathrm{~km}^{2}$, with an average elevation of $4200 \mathrm{~m}$ and a population of 42,000 (in 2006). The population comprised $98.8 \%$ Tibetans living in six pastoral communes and two townships. Domestic animals included goats, sheep, yaks and horses. The average annual temperature is $1.3^{\circ} \mathrm{C}$ (mean range -10.4 to $10.7^{\circ} \mathrm{C}$, greatest range -32.5 to $26.5^{\circ} \mathrm{C}$ ). Wild ungulates in Dangxiong County included Tibetan wild ass (Equus kiang), blue sheep (Pseudois nayaur) and yellow sheep (Procapra picticaudata). Common small mammals in Dangxiong County included plateau pika (Ochotona curzoniae), Himalayan marmot (Marmota himalayana), Tibetan hare (Lepus oiostolus) and voles (Microtus spp.).

Dingqing County (Tibetan: Dengqen or Temgchen), is one of 11 counties in Changdu Prefecture (also called Chamdo or Qamdo) located in the north-east of TAR, and adjoins Qinghai Province to the north and Sichuan and Yunnan provinces to the east. The average elevation of Dingqing County is $4000 \mathrm{~m}$, with an area of $11,365 \mathrm{~km}^{2}$ that includes mostly pasture with just 110,000 ha of agricultural land and $6.7 \%$ forest. The average annual rainfall is $641 \mathrm{~mm}$ and the average annual temperature $3.1^{\circ} \mathrm{C}$ (full range -25 to $27^{\circ} \mathrm{C}$ ). The population was approximately 62,500 of which $>99 \%$ were of Tibetan ethnicity. Most residents (99\%) worked as herdsmen or farmers in two townships and 65 villages of Dingqing County. Livestock in north-west Dingqing County were yak, cattle, yak-cattle cross-breeds, sheep, goats, horses, donkeys and mules. The main agriculture products were barley, wheat and peas from south-east Dingqing. In addition, about $60 \%$ of the population was involved with the annual spring collection from pasture topsoil of 'winter worm' or yartsa gunbu, a caterpillar fungus (Cordyceps sinensis) that is a highly valued traditional Chinese medicine. Domestic dogs (owned and stray) were very numerous around towns or villages, and there were approximately 1-3 dogs per household. Common wild mammals in the county included Tibetan fox (Vulpes ferrilata), red fox (Vulpes vulpes), wolf (Canis lupus), plateau pika (O. curzoniae), microtine voles (Microtus spp.), Himalayan marmots ( $M$. himalayana) and Tibetan woolly hares (L. oiostolus). 


\section{Tibet Autonomous Region}
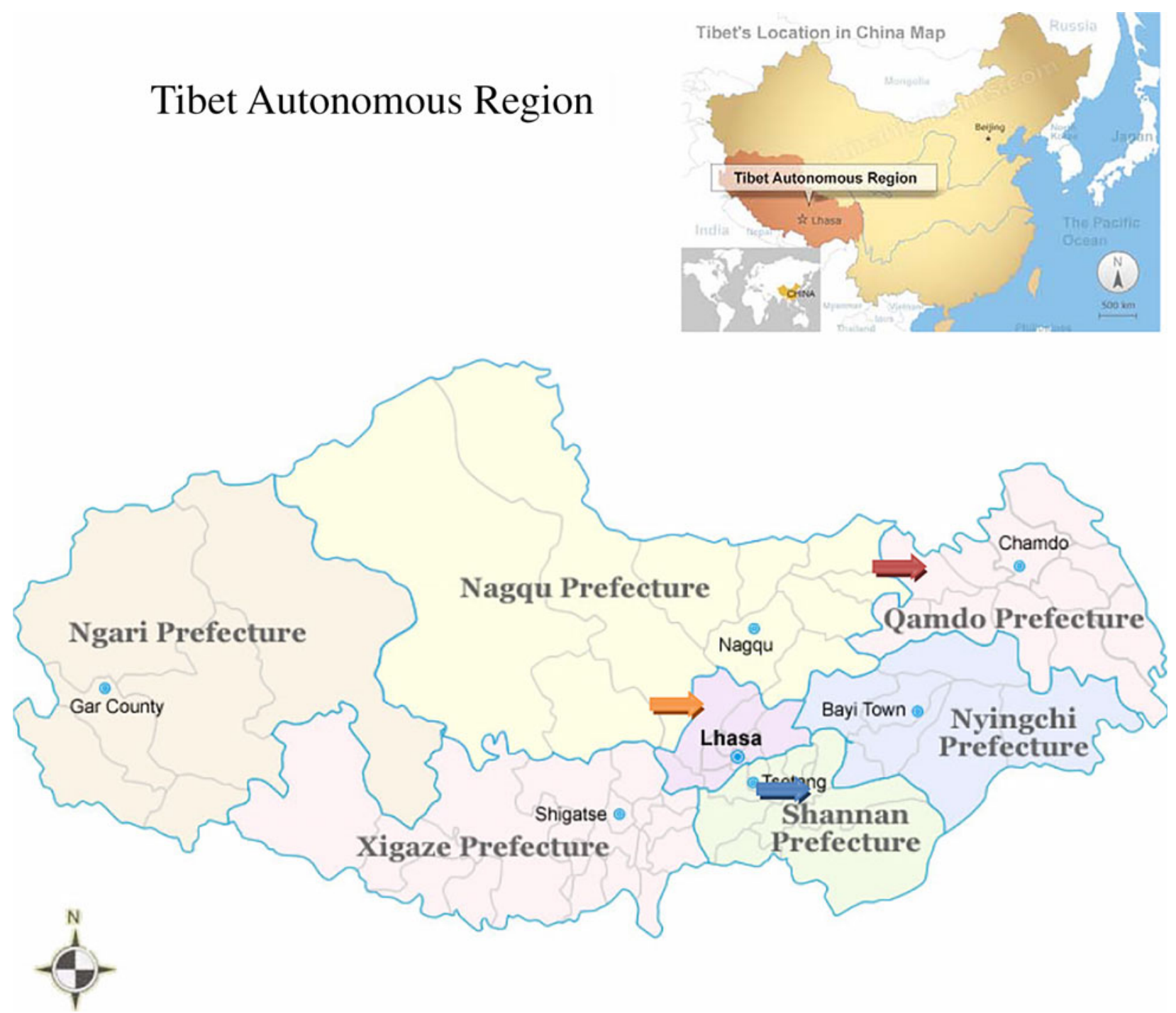

Fig. 1. The three counties in Tibet surveyed for human echinococcosis, using historical data from Qusong (blue arrow) in 2003 and ultrasound screening of communities in Dangxiong (orange arrow) in 2006 and Dingqing (red arrow) in 2007.

\section{Human screening}

Community surveys for human echinococcosis were undertaken based upon voluntary self-selection, and included a questionnaire, registration, ultrasound scanning and rapid serological testing using dot immunogold filtration assay (DIGFA) (Feng et al., 2010). All screened people agreed voluntarily to attend the survey and gave informed consent. The registration questionnaires included general information - name, ethnicity, sex, age, occupation, education level, annual income, etc. and were performed by local Tibetan-speaking Centre for Disease Control (CDC) staff for exact records.

In Dangxiong County, two communes (Wuma and Ningzhong) and one town (Yangbajin) were screened in October 2006 (total population approximately 18,000).
In total, 557 persons volunteered for screening, including 212 males and 345 females, 532 persons originated from the above areas and 25 from other communes (excluding Namco). In total, 488 people volunteered to donate $2-3 \mathrm{ml}$ venous blood for rapid serological test by DIGFA, and among those 165 were tested further by standard enzymelinked immunosorbent assay (ELISA) at Xinjiang Medical University Hospital (Urumqi) according to Feng et al. (2010). In Dingqing County, 232 people from three communes/towns (total population approximately $18,500)$ were screened in 2007 . In total $195 / 232$ persons donated $2-3 \mathrm{ml}$ venous blood for DIGFA sero-testing.

The volunteers were given an abdominal ultrasound scan (portable ultrasound GE LOGO XP, China) by experienced sonographers (L. Yang, B. Bartholomot). 
Ultrasound hepatic images were recorded as $\mathrm{CE}, \mathrm{AE}$, query, other lesions or normal. Those who had characteristic $\mathrm{CE}$ or $\mathrm{AE}$ ultrasound images or a query lesion were recorded. All CE or AE cases confirmed in the survey were offered a free 6-month course of albendazole treatment. Cases of CE diagnosed in Dangxiong were treated surgically (cystectomy) in the People's Hospital of Lhasa City by surgeons from Xinjiang Medical University Hospital with the cooperation of surgeons from the People's Hospital of Lhasa City.

\section{Serology}

A rapid DIGFA developed by Feng et al. (2010), available in kits (from Xinjiang Bestmind Biological Technique Development Ltd, Urumqi, China), was performed within 1 or $2 \mathrm{~h}$ after ultrasound scan. Blood samples were centrifuged at $2000 \mathrm{~g}$ after $1 \mathrm{~h}$ (if using general blood collection tubes) or $10 \mathrm{~min}$ (if using procoagulant tubes) and sera were tested immediately. DIGFA used four native antigen preparations: crude E. granulosus cyst fluid antigen (EgCF), E. granulosus protoscoleces extract $(\mathrm{EgP}), E$. granulosus cyst fluid antigen $\mathrm{B}(\mathrm{AgB})$ and $E$. multilocularis laminated layer antigen (Em2) (Feng et al., 2010). If either one of the EgCF, $\mathrm{EgP}, \mathrm{AgB}$ or Em2 spots appeared (red), the sera were determined to be positive for echinococcosis; otherwise, if none appeared, the sera were considered negative. Positive, query or negative spots were recorded for the four antigens respectively. Positives with any one of four antigens were recognized as sero-positive. Results positive with $\mathrm{AgB}$ and negative or weakly positive with Em2 (e.g. antigen $B$ ' + ' and Em2 ' - ' or ' \pm ') were indicative of antibodies to CE. Positives against Em2 and negative or weakly positive with $\mathrm{AgB}$ (e.g. Em2 ' $++{ }^{\prime}$ and antigen $\mathrm{B}$ ' $-{ }^{\prime},{ }^{\prime} \pm$ ' or ' + ') were recognized as indicative of the presence of antibodies to $\mathrm{AE}$ (Feng et al., 2010).

In addition to serum samples collected in association with the active ultrasound-based screening in Dangxiong and Dingqing counties, a panel of archived sera was available from an earlier ultrasound survey (2003, $N=722$ ) carried out in Qusong County (Shannan Prefecture) in south-central TAR (fig. 1). Archived sera were tested by DIGFA and matched to respective ultrasound results, but without personal details and questionnaire information. Serological positives and ultrasound-confirmed $\mathrm{CE}$ or $\mathrm{AE}$ cases were recorded and followed up as above.

\section{Review of published CE and AE reports for TAR (1980-2007)}

Scientific papers on echinococcosis in Tibet Autonomous Region were identified in a Chinese academic search engine (CNKI, Wanfang and VIP) for the Chinese publications, as well as in the Medline/Pubmed search engine for international publications.

\section{Data analysis}

Data from questionnaires, ultrasound and serological results were analysed using SPSS 16.0 (SPSS Inc., Chicago, Illinois, USA).

\section{Results}

A search of published studies from Chinese and international literature (1980-2007) relating to human echinococcosis in the TAR identified 27 publications, of which 15 were most relevant.

Reports confirmed that human CE (total cases $n=1641)$ has been recorded in all seven prefectures in

Table 1. Human cystic (CE) and alveolar (AE) echinococcosis recorded from hospital cases in the Tibet Autonomous Region, P.R. China from 1960 to 2007; $N=$ number of cases examined.

\begin{tabular}{|c|c|c|c|c|c|c|c|}
\hline \multicolumn{4}{|c|}{$\mathrm{CE}$} & \multicolumn{4}{|c|}{$\mathrm{AE}$} \\
\hline Lhasa & $1996-2000$ & 35 & Gong et al., 2001 & Naqu & & & \\
\hline \multirow[t]{3}{*}{ Changdu } & & 94 & Luo \& Zhao, 1994 & Shannan & & & \\
\hline & 1988-1995 & 70 & Lan et al., 1999 & Changdu & & & \\
\hline & $1996-2000$ & 10 & Gong et al., 2001 & & & & \\
\hline \multirow{5}{*}{ Shannan } & 1995-2001 & 80 & Zhou et al., 2002 & & 2006 & 4 & Hospital CT images \\
\hline & 1995-2004 & 116 & Peng et al., 2004 & & & & \\
\hline & $1988-2002$ & 268 & Liao et al., 2003 & & & & \\
\hline & $1999-2006$ & 59 & $\mathrm{Yu}, 2006$ & & & & \\
\hline & & 155 & Cai et al., 2007 & & & & \\
\hline Linzhi & $1996-2000$ & 8 & Gong et al., 2001 & Linzhi & 2006 & 3 & Duan et al., 2006 \\
\hline
\end{tabular}


the TAR (table 1$)$. In contrast, human AE cases $(n=22)$ were recorded only in Lhasa and Naqu prefectures and two eastern regions (Changdu and Linzhi prefectures) of TAR (table 1). One of the earliest reports of echinococcosis in the People's Hospital of Tibet AR (in Lhasa city) included $174 \mathrm{CE}$ cases and one $\mathrm{AE}$ case for the period 1960-1983 but without details of case origins (Hu et al., 1987). In the past 20 years, most human CE cases were recorded in prefectural hospitals in Naqu $(n=623)$, Shannan $(n=268)$ and Changdu $(n=182)$. For Lhasa, 174 $\mathrm{CE}$ and $162 \mathrm{CE}$ cases were reported during the period 1960-1988 from the two main public hospitals and, in addition, $80 \mathrm{CE}$ cases from a military hospital in Lhasa (Hu et al., 1987; Ciren \& Wang, 2000; Gong et al., 2001; Zhou et al., 2002; Liao et al., 2003; Peng et al., 2004; Yu, 2006; Cai et al., 2007). Human CE cases were also reported in Rikaze, Linzhi and Ali prefectures in western TAR, but numbers were small (seven hepatic and two uterine CE cases in Rikaze, and 18 hepatic CE cases with computed tomography (CT) scan confirmation in Linzhi Prefecture Hospital) (Hou et al., 2005; Duan et al., 2006) (table 1).

Reports of human AE cases have been sporadic in TAR, in contrast to Tibetan communities in neighbouring Sichuan and Qinghai provinces to the east and north-east of TAR but still situated on the Tibetan Plateau (Li et al., 2005, 2010). After a first reported AE case in 1987, other reports showed two cases from Changdu (Peng, 1988), 12 cases from Lhasa, Naqu and Changdu prefectures (Yixi, 1992; Luo \& Zhao, 1994), and three AE cases diagnosed by CT scanning in Linzhi (Duan et al., 2006) (table 1).

We undertook a retrospective search of local patient records at the Changdu Prefecture Hospital for the period 2001-2005 and identified 61 CE cases and four putative AE cases. From those records $17 \mathrm{CE}$ and three AE cases were confirmed to reside in Dingqing County in eastern TAR.

Previous mass ultrasound screenings in communities have also detected $48 \mathrm{CE}$ cases in Lhasa Prefecture and nine CE cases in Qusong County of Shannan Prefecture (Hu et al., 1999; unpublished study in 2003). No AE cases were specifically identified in these previous community studies in TAR. In the current study, pilot ultrasound screening (combined with rapid serology) was undertaken in Dangxiong County (Lhasa Prefecture) and Dingqing County (Changdu Prefecture). Data were also available from an earlier mass screening programme in Qusong County (table 2). The prevalence of human CE by ultrasound was $9.9 \%(55 / 557)$ in Dangxiong, $4.7 \%$ $(11 / 232)$ in Dingqing and $1.2 \%(9 / 722)$ in Qusong (table 2). Human AE cases were only confirmed in the Dingqing survey but with an apparently high prevalence of $5.2 \%(12 / 232)$ including one mixed AE/CE case (table 2). Two query $\mathrm{AE}$ cases were, however, also listed from the Qusong survey. Sero-testing gave sero-prevalences $>6 \%$ in Qusong and Dangxiong, but a significantly higher sero-prevalence (35.4\%) for the Dingqing survey (chi-square $P<0.001$ ) (table 2).

The incidence of human $\mathrm{CE}$ was estimated from these hospital records for the seven prefectures of TAR, and ranged from 1.9 to 155 per 100,000, with a mean incidence throughout TAR of 45.1 per 100,000 (table 3). Human AE incidence was based on smaller datasets but was estimated to range between 0.6 and 2.8 per 100,000 , with the highest incidence in Changdu Prefecture in eastern TAR.

\section{Discussion}

Previous studies have identified very high prevalences of both $\mathrm{CE}$ and $\mathrm{AE}$ in Tibetan communities in western Sichuan and south-eastern Qinghai provinces of China (outside the TAR), with mean prevalences of up to $15 \%$ at the township level for both $\mathrm{CE}$ and AE. These prevalences are among the highest in the world. However, despite having a considerable Tibetan population, there is little data about CE, and especially AE, in the TAR itself.

Hospital records in TAR listed 709 cases of human CE from three hospitals in Naqu Prefecture for the period 1996-2000 (Gong et al., 2001). In that study 623 CE cases were originally from Naqu Prefecture and the other CE cases were from the prefectures of Lhasa $(n=35)$, Shannan $(n=22)$, Changdu $(n=10)$, Linzhi $(n=8)$, Rikaze $(n=4)$ and Ali $(n=2)$, and other areas $(n=5)$ (Gong et al., 2001). A total of 94 cases of echinococcosis (not differentiated as $\mathrm{CE}$ or $\mathrm{AE}$ ), were reported in Changdu Prefecture (Luo \& Zhao, 1994). In Shannan Prefecture 116 (period 1995-2004), 155 (to 2007) and 268 (1988-2002) echinococcosis cases were reported (Liao et al., 2003; Peng et al., 2004; Cai et al., 2007).

Most of the mass screening community studies in TAR to date have used the relatively non-specific Casoni skin test (the prevalence based on this test was estimated as $34.9 \%$ over the period 1988-1991 (Guo \& Yu, 1994)). One survey in Dangxiong and Mozugongka counties of Lhasa Prefecture, confirmed $48 \mathrm{CE}$ cases from 734 individuals testing positive for the Casoni skin test in a test population of 20,160 (Hu et al., 1999). Mass screening

Table 2. The prevalence (\%) of human cystic (CE) and alveolar (AE) echinococcosis screened by ultrasound or serology in Qusong, Dangxiong and Dingqing counties, TAR from 2003 to 2007; $N=$ number of cases examined.

\begin{tabular}{lcccccr}
\hline & & & & & \multicolumn{2}{c}{ Sero-positivity } \\
\cline { 5 - 7 } Counties & $N$ & CE (\%) & AE (\%) & CE/AE (\%) & $N$ & Positives (\%) \\
\hline Qusong & 722 & $9(1.2)$ & 0 & 0 & 722 & $55(7.6)$ \\
Dangxiong & 557 & $55(9.9)$ & 0 & 0 & 488 & $31(6.4)$ \\
Dingqing & 232 & $11(4.7)$ & $11(4.7)$ & $1(0.4)$ & 195 & $69(35.4)$ \\
Total & 1511 & $75(4.96)$ & 11 & 1 & 1405 & $155(11.0)$ \\
\hline
\end{tabular}


Table 3. Estimated incidences (per 100,000) and confirmed cases of human cystic (CE) and alveolar (AE) echinococcosis in TAR, relative to prefecture from 1960 to 2005.

\begin{tabular}{lcccccc}
\hline & & \multicolumn{2}{c}{ CE } & & \multicolumn{2}{c}{ AE } \\
\cline { 3 - 4 } \cline { 6 - 7 } Prefecture & $\begin{array}{c}\text { Population } \\
\text { (2005) }\end{array}$ & Confirmed & Incidence & & Confirmed & Incidence \\
\hline Lhasa & 540,500 & 138 & 25.5 & & \multirow{2}{*}{0.6} \\
Naqu & 401,871 & 623 & 155.0 & & & \\
Ali & 86,277 & 2 & 2.3 & & \\
Rikaze & 662,146 & 13 & 1.96 & & \\
Linzhi & 158,167 & 26 & 16.4 & & 3 & \\
Shannan & 325,063 & 268 & 82.4 & & & \\
Changdu & 606,444 & 182 & 30.0 & & 17 & 0.8 \\
Tibet AR & $2,778,463$ & 1252 & 45.1 & & 23 & \\
\hline
\end{tabular}

using portable ultrasound scanning for abdominal CE (or AE) is a much more specific approach (Bartholomot et al., 2002; Macpherson et al., 2003; Li et al., 2005).

Overall, human AE is a rare disease at the national level in China; however, significant foci of $\mathrm{AE}$ have been described in central, north-west and south-west China (Craig et al., 1992; Zhou et al., 2000; Li et al., 2010). However, an important question remains: What is the extent of human AE within the TAR? This is pertinent especially because of the high prevalences of human $\mathrm{AE}$ detected in Tibetan communities on the eastern Tibetan plateau (but outside the TAR) in Sichuan and Qinghai provinces (Schantz et al., 2003; Li et al., 2010; Giraudoux et al., 2013b). The first human AE case reported in TAR was in 1987 in the TAR People's Hospital, Lhasa (Hu et al., 1987). In 1988 two AE cases (history identified from 1977) were reported from the eastern TAR in Changdu Prefecture (Peng, 1988). Since then, it appears that 12 $\mathrm{AE}$ cases were confirmed in hospitals in Lhasa, although their origins not only included cases from Lhasa Prefecture, but also from Naqu and Changdu prefectures (Pu, 1999; Yixi et al., 2001). Later, a further three AE cases, confirmed by CT scanning, were reported in Linzhi Prefecture (Duan et al., 2006). However, no AE cases were previously identified during mass screening community studies in TAR prior to the current study.

The objectives of the current study not only included a review of published literature relating to $\mathrm{CE} / \mathrm{AE}$ in TAR, and also of patient records in the People's Hospital of Changdu Prefecture (TAR), but in addition to undertake pilot community studies in two counties: Dangxiong in Lhasa Prefecture, central TAR, and Dingqing in Changdu Prefecture, situated in eastern TAR. Community surveys included questionnaire registration, abdominal ultrasound scanning and DIGFA sero-testing. In addition, ultrasound records from an unpublished community study carried out in 2003 in Qusong County (Shannan Prefecture) were examined in detail. In these three pilot ultrasound-based community mass screenings, AE cases were only detected in Dingqing County (Changdu Prefecture) but with a high prevalence of $5.2 \%$ in the screened population (including a case of mixed $\mathrm{AE} / \mathrm{CE}$ infection). No AE cases were identified in the 1279 people screened in Dangxiong and Qusong counties. In contrast, human CE cases have been found in all counties where mass screening was performed and have been treated in all seven prefectures of TAR (Gong et al., 2001), with most hospital cases in Naqu, Shannan, Changdu, Lhasa and Linzhi prefectures. This supports previous community studies by the Chinese Ministry of Health (Ministry of Health, 2005), which found that human CE cases were located mainly in the south-east, east and north-central areas of TAR (table 3). Previous reports showed that the highest ultrasound prevalence for human CE in China occurred in Tibetan pastoral communities of the eastern Tibetan plateau, including south-west Qinghai and northwest Sichuan provinces (Qiu \& Wang, 1999; Schantz et al., 2003; Craig, 2004; Li et al., 2010; Giraudoux et al., 2013b). In the current pilot study in TAR we determined the point prevalence of ultrasound-diagnosed human CE as 9.9\% (55/557) in Dangxiong County, and 4.7\% (11/232) in Dingqing County; in addition, a review of ultrasound records from a prior screening in 2003 in Qusong County provided a CE prevalence of 1.2\% (9/722). The prevalence of $C E$ was significantly higher for Dangxiong $(P<0.01)$. Human CE incidence for the whole of TAR was estimated to be 45.1 per 100,000 , with a range of 1.9 to 155 per 100,000 and the highest incidence in Naqu Prefecture. However, it is important to consider the limitations in these estimates, partly as a result of the challenges in estimating the incidence of an infection with such a long latent period before clinical signs manifest, and partly due to the biases associated with the use of hospital records rather than statistically sound surveys.

Transmission of E. granulosus in TAR is almost certainly related to the widespread distribution and high density of both dogs and domestic livestock (sheep, goats, yak) (Eckert et al., 2001; Craig, 2004), especially in central and eastern areas (Giraudoux et al., 2013a). Echinococcus granulosus was recorded from the intestines of dogs in Naqu (TAR), and was reported to be common in yak, cattle, sheep and goats in TAR (Zhang et al., 1994; Nima, 1998; Lan et al., 1999). The transmission ecology of E. multilocularis in TAR is not known, but will probably be similar to that described in Tibetan autonomous regions in north-west Sichuan and south Qinghai, i.e. a wildlife cycle involving Tibetan foxes ( $V$. ferrilata) and a number of microtine and/ or ochotonid small mammal species (Raoul et al., 2006). Furthermore, the role of domestic dogs in the transmission of human AE in Tibetan communities in TAR is likely to be similar to that described in Tibetan communities in northwest Sichuan (Vaniscotte et al., 2011; Giraudoux et al., 2013a; 
Moss et al., 2013; Wang et al., 2015). The distribution of human AE disease in western China was found to be related to the proportion of alpine meadows / grasslands in a given region, which provide optimal habitats for potential small mammal intermediate hosts (Giraudoux et al., 2013a). Co-occurrence of both $\mathrm{CE}$ and $\mathrm{AE}$ in the same patient, as identified in Dingqing County, would support the role of dogs in transmission. Such double infection has been identified at least twice in other regions of China (Ningxia and Xinjiang) (Wen et al., 1992; Yang et al., 2006), and double intestinal infection of a dog with E. granulosus and E. multilocularis has been demonstrated using molecular tools (Zhang et al., 2006). Furthermore the 'landscape risk model' of Giraudoux et al. (2013b) predicted two human AE transmission hotspots in eastern TAR. One was in Dingqing, which appears to be confirmed by the current study, the other was to the north-west of Naqu, close to the border with Qinghai Province; however, to date no community screening has been reported from the latter area.

In summary, both human cystic echinococcosis (CE) and alveolar echinococcosis (AE) are endemic in the Tibet Autonomous Region (TAR). Human CE is relatively widespread, occurring in all of the seven prefectures of TAR, but with higher case numbers, incidence and prevalence in central and eastern areas of TAR. CE incidence was estimated to be 45.1 per 100,000 for the TAR. In contrast, fewer than 20 human AE cases have been reported from hospitals in central and eastern prefectures; however, a pilot mass screening using ultrasound revealed a significant $\mathrm{AE}$ prevalence $(5.2 \%)$ in Dingqing County in the eastern prefecture of Changdu. It is anticipated that extended mass screening will detect more cases of human AE in the eastern region of TAR.

\section{Acknowledgements}

We wish to acknowledge the excellent assistance of staff from the regional Centres for Disease Prevention and Control (CDC) of Lhasa, Changdu and Shannan prefectures in TAR.

\section{Financial support}

This work was mainly supported by a Program for Changjiang Scholars and Innovative Research Team in University (PCSIRT) from the Ministry of Education of P.R. China ('Echinococcus Pathogenesis and Integrated Control Research', grant \#IRT1181), funding from the Chinese State Key Laboratory Incubation Base of Xinjiang Major Diseases Research (lab \#2010DS890294) (Urumqi, China), funding from The First Affiliated Hospital of Xinjiang Medical University (2011YFY16) and in part by a project grant from the Wellcome Trust (UK) (grant \#094325/Z/10/Z).

\section{Conflict of interest}

None.

\section{Ethical standards}

The study was approved by the ethics committee at the First Affiliated Hospital of Xinjiang Medical University,
Urumqi, P.R. China. All patients' data in the archives of hospitals were kept private and anonymous using a coding system for patients' names.

\section{References}

Bai, Y.N., Cheng, N., Jiang, C.P., Wang, Q. \& Cao, D.R. (2002) Survey on cystic echinococcosis in Tibetans, West China. Acta Tropica 82, 381-385.

Bartholomot, G., Vuitton, D.A., Harraga, S., Shi, D.Z., Giraudoux, P., Barnish, G., Wang, Y.H., Macpherson, C.N.L. \& Craig, P.S. (2002) Combined ultrasound and serologic screening for hepatic alveolar echinococcosis in central China. American Journal of Tropical Medicine and Hygiene 66, 23-29.

Budke, C.M., Campos-Ponce, M., Wang, Q. \& Torgerson, P.R. (2005) A canine purgation study and risk factor analysis for echinococcosis in a high endemic region of the Tibetan plateau. Veterinary Parasitology 127, 43-49.

Cai, Z., Peng, S., Shen, D., Yan, M. \& Xi, Q. (2007) Experience of surgical treatment for 155 cases of hepatic echinococcosis. Journal of Military Surgeon in Southwest (China) 9, 66-67.

Ciren, D. \& Wang, H. (2000) Report on surgical treatment of 80 cases of hepatic echinococcosis. Medical Journal of the Chinese People's Armed Police Forces (Wu Jing Yi Xue) 11, 548.

Craig, P.S. (2004) Epidemiology of echinococcosis in China. Southeast Asian Journal of Tropical Medicine and Public Health 35 (Suppl. 1), 158-169.

Craig, P.S., Liu, D., Shi, D., Macpherson, C.N.L., Barnish, G., Reynolds, D., Gottstein, B. \& Wang, Z. (1992) A large focus of alveolar echinococcosis in central China. Lancet 340, 826-831.

Craig, P.S., McManus, D.P., Lightowlers, M.W., Chabalgoity, J.A., Garcia, H.H., Gavidia, C.M., Gilman, R.H., Gonzalez, A.E., Lorca, M., Naquira, C., Nieto, A. \& Schantz, P.M. (2007) Prevention and control of cystic echinococcosis. Lancet Infectious Diseases 7, 385-394.

Craig, P.S., Li, T.Y., Qiu, J.M., Zhen, R., Wang, Q., Giraudoux, P., Ito, A., Heath, D.D., Warnock, B. \& Schantz, P.M. (2008) Echinococcoses and Tibetan communities. Emerging Infectious Diseases 14, $1674-1675$.

Duan, X., Zhajie, Yongqing, B. \& Shang, L. (2006) SCT diagnosis of hepatic hydatid disease. Journal of Practical Medical Techniques (Shi Yong Yi Ji Za Zhi) 13, 2399-2400.

Eckert, J., Gemmell, M.A., Meslin, F.-X., Pawlowski, Z.S. (Eds) (2001) WHO/OIE manual on echinococcosis in humans and animals: A public health problem of global concern. Paris, World Health Organization/Organization for Animal Health.

Feng, X., Wen, H., Zhang, Z., Chen, X., Ma, X., Zhang, J., Qi, X., Bradshaw, H., Vuitton, D.A. \& Craig, P.S. (2010) Dot immunogold filtration assay (DIGFA) with multiple native antigens for rapid serodiagnosis of human cystic and alveolar echinococcosis. Acta Tropica 113, 114-120.

Giraudoux, P., Raoul, F., Afonso, E., Ziadinov, I., Yang, Y., Li, L., Li, T., Quéré, J.P., Feng, X., Wang, Q., Wen, H., Ito, A. \& Craig, P.S. (2013a) Transmission ecosystems 
of Echinococcus multilocularis in China and Central Asia. Parasitology 140, 1655-1666.

Giraudoux, P., Raoul, F., Pleydell, D., Li, T., Han, X., Qiu, J., Xie, Y., Wang, H., Ito, A. \& Craig, P.S. (2013b) Drivers of Echinococcus multilocularis transmission in China: small mammal diversity, landscape or climate? PLoS Neglected Tropical Diseases 7, e2045.

Gong, X., Ciwang, R., Ze, Y., Lu, H., Pan, X. \& Weng, X. (2001) Clinical analysis for 709 cases of cystic echinococcosis in Tibet Autonomous Region. Chinese Journal of Parasitology $\mathcal{E}$ Parasitic Diseases (Zhong Guo Ji Sheng Chong Xue Yu Ji Sheng Chong Bing Za Zhi) 19, 128.

Guo, W. \& Yu, D. (1994) Investigation on the distribution of human parasites in Tibet Autonomous Region. Chinese Journal of Parasitic Disease Control (Zhong Guo Ji Sheng Chong Bing Fang Zhi Za Zhi) 7, 131-132.

Hou, J., Zhaxi, C. \& Yixi, Q. (2005) Hydatidosis in female reproductive organ (report of 9 cases). Journal of Chinese Modern Obstetrics and Gynecology 2, 271.

Hu, R., Luobu, W., Li, M., Chang, J., Chen, Q., Qiongda, G., Siqu, G.Q., Danzeng, O., Quzha, S.W., Cangjue, Y.Z., Sangzhen, Q.X., Laba, Z.M., Dai, X. \& Selimi \& Hao, J. (1999) Sampling survey of echinococcosis infection and state of illness in pastoral area of Tibet. Tibetan Journal of Medicine (Xi Zang Yi Yao Za Zhi) 20, 11-14.

Hu, X., Luozhu, Q., Jiang, M. \& Hu, J. (1987) Clinical assessment of 175 cases of echinococcosis in Tibet Autonomous Region. Chinese Journal of Zoonosis (Zhong Guo Ren Shou Gong Huan Bing Za Zhi) 3, 57-59.

Jiang, C. (2002) Today's regional distribution of echinococcosis in China. Chinese Medical Journal (English) 115, 1244-1247.

Lan, S., Gasong, D. \& Baima, L. (1999) Investigation of echinococcosis in human and livestock in Changdu Prefecture, Tibet Autonomous Region. Chinese Journal of Veterinary Parasitology (Zhong Guo Shou Yi Ji Sheng Chong Bing) 7, 23-24.

Li, T., Qiu, J., Yang, W., Craig, P.S., Chen, X., Xiao, N., Ito, A., Giraudoux, P., Mamuti, W., Yu, W. \& Schantz, P.M. (2005) Echinococcosis in Tibetan populations, Western Sichuan Province, China. Emerging Infectious Diseases 11, 1866-1873.

Li, T., Chen, X., Zhen, R., Qiu, J., Qiu, D., Xiao, N., Ito, A., Wang, H., Giraudoux, P., Sako, Y., Nakao, M. \& Craig, P.S. (2010) Widespread co-endemicity of human cystic and alveolar echinococcosis on the eastern Tibetan Plateau, northwest Sichuan/southeast Qinghai, China. Acta Tropica 113, 248-256.

Liao, L., Peng, S., Wang, X., Feng, G., Hu, Z. \& Zhou, Z. (2003) Investigation of 268 cases of hydatidosis in area of elevation $3700 \mathrm{~m}$. Journal of High Altitude Medicine (Gao Yuan Yi Xue Za Zhi) 4, 50-51.

Luo, S. \& Zhao, Y. (1994) Ultrasound diagnosis on 94 cases of liver echinococcosis in Changdu Prefecture. Tibetan Journal of Medicine (Xi Zang Yi Yao Za Zhi) 6, 62-63.

Luo, Y. \& Peng, S. (1993) Analysis of 12 cases of hepatic alveolar echinococcosis in Tibet. Chinese Journal of Parasitic Disease Control (Zhong Guo Ji Sheng Chong Bing Fang Zhi Za Zhi) 6, 62-63.

Macpherson, C.N.L., Bartholomot, B. \& Frider, B. (2003) Application of ultrasound in diagnosis, treatment, epidemiology, public health and control of Echinococcus granulosus and E. multilocularis. Parasitology 127, S21-S35.

Ministry of Health, P.R. China. (2005) Report on the national survey of current situation of major human parasitic diseases in China. Shanghai, National Institute of Parasitic Diseases.

Moss, J.E., Chen, X., Li, T., Qiu, J., Wang, Q., Giraudoux, P., Ito, A., Torgerson, P.R. \& Craig, P.S. (2013) Reinfection studies of canine echinococcosis and role of dogs in transmission of Echinococcus multilocularis in Tibetan communities, Sichuan, China. Parasitology 140, 1685-1692.

National Bureau of Statistics of China, (2007) China statistical yearbook. Beijing, China Statistics Press.

Nima, C. (1998) Investigation of animal parasite disease in Ali Prefecture, Tibet. China Animal Husbandry Bulletin (Zhong Guo Mu Ye Tong Xun) 8, 19.

Peng, S. (1988) Report of two alveolar echinococcosis cases from Changdu Prefecture, Tibet Autonomous Region. Chinese Journal of Parasitology and Parasite Disease (Zhong Guo Ji Sheng Chong Xue Yu Ji Sheng Chong Bing Za Zhi) 6, 288.

Peng, S., Xu, Q. \& Zhou, Z. (2004) Cyst puncture aspiration in 40 cases of abdominal echinococcosis. Academic Journal of the First Medical College of PLA (Di 1 jun yi da xue xue bao) 24, 1333-1334.

Pu, Z. (1999) A case of brain alveolar echinococcosis. Chinese Journal of Neurosurgery (Zhong Hua Shen Jing Wai Ke Za Zhi) 6, 338.

Qiu, J. \& Wang, Y. (1999) Analysis on 4486 cases of hydatid disease in a hospital in past 41 years. Chinese Journal of Health Statistics (Chinese) 16, 168-169.

Raoul, F., Quere, J.-P., Rieffel, D., Bernard, N., Takahashi, K., Scheifler, R., Ito, A., Wang, Q., Qiu, J., Yang, W., Craig, P.S. \& Giraudoux, P. (2006) Distribution of small mammals in a pastoral landscape of the Tibetan plateaus (western Sichuan, China) and relationship with grazing practices. Mammalia 214-225.

Schantz, P.M., Wang, H., Qiu, J., Liu, F.J., Saito, E., Emshoff, A., Ito, A., Roberts, J.M. \& Delker, C. (2003) Echinococcosis on the Tibetan Plateau: prevalence and risk factors for cystic and alveolar echinococcosis in Tibetan populations in Qinghai Province, China. Parasitology 127, S109-S120.

Vaniscotte, A., Raoul, F., Poulle, M.L., Romig, T., Dinkel, A., Takahashi, K., Guislain, M.H., Moss, J., Li, T., Wang, Q., Qiu, J., Craig, P.S. \& Giraudoux, P. (2011) Role of dog behaviour and environmental fecal contamination in transmission of Echinococcus multilocularis in Tibetan communities. Parasitology 138, 1316-1329.

van Kesteren, F., Qi, X., Tao, J., Feng, X., Mastin, A., Craig, P.S., Vuitton, D.A., Duan, X., Chu, X., Zhu, J. \& Wen, H. (2015) Independent evaluation of a canine echinococcosis control programme in Hobukesar County, Xinjiang, China. Acta Tropica 145, 1-7.

Wang, Q., Yu, W., Zhong, B., Shang, J., Huang, L., Renqingpengcuo, Huang, Y., Zhang, G., He, W., Giraudoux, P. \& Craig, P.S. (2015) Seasonal pattern of Echinococcus spp. re-infection in dogs in Tibetan 
communities and its implications for control, Sichuan, China. Journal of Helminthology.

Wen, H., Tian, W.L., Zou, P.F. \& Xiang, M.X. (1992) A rare case of mixed cystic and alveolar hydatidosis. Transactions of the Royal Society of Tropical Medicine and Hygiene 86, 290-291.

Yang, Y.R., Liu, X.Z., Vuitton, D.A., Bartholomot, B., Wang, Y.H., Ito, A., Craig, P.S. \& McManus, D.P. (2006) Simultaneous alveolar and cystic echinococcosis of the liver. Transactions of the Royal Society of Tropical Medicine and Hygiene 100, 597-600.

Yin, C. \& Wang, L. (2007) The diagnostic value of routine examinations of hydatid disease. Tibetan Journal of Medicine (Xi Zang Yi Yao Za Zhi) 28, 54-55.

Yixi, J. (1992) Pathological analysis of 10 cases of alveolar echinococcosis in Tibet Autonomous Region. Tibetan Journal of Medicine (Xi Zang Yi Yao Za Zhi) 13, 14-15.

Yixi, J., Sui, G. \& Quzhen (2001) A case report of secondary alveolar echinococcosis in brain. Chinese Journal of Pathology (Zhong Hua Bing Li Xue Za Zhi) 30, 349.
Yu, C. (2006) Ultrasound diagnosis analysis for 59 cases of hepatic echinococcosis. People's Military Surgeon (Ren Min Jun Yi) 49, 410-411.

Zhang, Y., Bart, J.-M., Giraudoux, P., Craig, P., Vuitton, D. \& Wen, H. (2006) Morphological and molecular characteristics of Echinococcus multilocularis and Echinococcus granulosus mixed infection in a dog from Xinjiang, China. Veterinary Parasitology 139, 244-248.

Zhou, H.X., Chai, S.X., Craig, P.S., Delattre, P., Quere, J.P., Raoul, F., Vuitton, D.A., Wen, H. \& Giraudoux, P. (2000) Epidemiology of alveolar echinococcosis in Xinjiang Uygur autonomous region, China: a preliminary analysis. Annals of Tropical Medicine and Parasitology 94, 715-729.

Zhou, Z., Li, D., Peng, S., Yan, C., Yan, M., Yu, M. \& Chang, Q. (2002) Vesiclectomy for patients of hepatic echinococcosis in Tibet: a report of 80 cases. Chinese Journal of General Surgery (Zhong Hua Pu Tong Wai Ke Za Zhi) 17, 551-552. 